\title{
ZOLEDRONIC ACID: A MISCHIEVOUS SUSPECT FOR LIVER INJURY
}

\author{
RUPAM GILL ${ }^{1}$, BALAJI 0 ${ }^{1}$, MEENA KUMARI K ${ }^{*}$, AMBERKAR MOHAN BABU V1 ${ }^{1}$, KARTHIK S UDUPA ${ }^{2}$
}

${ }^{1}$ Department of Pharmacology, Kasturba Medical College, Manipal University, Manipal - 576 104, Karnataka, India. ${ }^{2}$ Department of Medical Oncology, Kasturba Hospital, Kasturba Medical College, Manipal University, Manipal - 576 104, Karnataka, India. Email: mini41178@yahoo.co.in

Received: 10 June 2016, Revised and Accepted: 23 June 2016

\section{ABSTRACT}

A 47-year-old male diagnosed as adenocarcinoma of the lung and received 8 cycles of chemotherapy comprising intravenous administration of cisplatin $125 \mathrm{mg}$, pemetrexed $850 \mathrm{mg}$ along with zoledronic acid $4 \mathrm{mg}$. After the completion of the $8^{\text {th }}$ cycle, the liver enzymes were found to be markedly elevated, evincing zoledronic acid as the cause for hepatotoxicity. The case details were taken from the patient's medical record along with the biochemical test reports and radiographic images. The causal association was confirmed using Naranjo's algorithm and Roussel Uclaf Causality Assessment Method (RUCAM). After the uneventful chemotherapy, patient's liver function tests (LFT) were abnormal. There was an elevation in the aspartate aminotransferase, alanine transaminase, alkaline phosphatase, and direct bilirubin. The causal relationship was established using Naranjo's algorithm (score-6) and RUCAM (score-5), displayed a "probable" and "possible" association. Hartwig's severity scale and Thornton's preventability scale displayed the adverse drug reaction to being moderately severe and not preventable, respectively. The zoledronic acid was stopped and never readministered. The LFTs assumed normal after a span of 2 months. The mechanism underlying hepatotoxicity due to zoledronic acid remains elusive. Zoledronic acid can induce acute phase response mediated by active production of interleukin-6, tumor necrosis factor alpha, and pro-inflammatory cytokines from the T-cells and macrophages. Vigilant monitoring along with timely assessment and management can prevent the occurrence of irreversible liver damage. Henceforth, we would like to report the rare incidence of drug induced hepatic damage due to zoledronic acid. Henceforth, we would like to report the rare incidence of drug induced hepatic damage due to zoledronic acid.

Keywords: Bisphosphonate, Dechallenge, Hepatotoxicity, Rechallenge.

(c) 2016 The Authors. Published by Innovare Academic Sciences Pvt Ltd. This is an open access article under the CC BY license (http://creativecommons. org/licenses/by/4. 0/) DOI: http://dx.doi.org/10.22159/ajpcr.2016.v9s2.13412

\section{INTRODUCTION}

Cancers are the leading cause of mortality worldwide with the most common cancers responsible for half of the global incidence is lung, breast, colorectal, prostate, stomach, and liver. Worldwide among men, lung cancer is more prevalent, whereas breast cancer typically predominates amid women. Overall, lung cancer is the most commonly diagnosed cancer worldwide with about 1.8 million new cases diagnosed every year with 1.6 million deaths [1]. Every year in India around 63,000 new lung cancer cases are reported [2]. Among the various histological subtypes of lung cancer, trends show increased incidence of adenocarcinoma among both men and women [3]. Pemetrexed-containing chemotherapeutic regimens are now widely used compared to taxanes, platinum-based, and other chemotherapeutic agents [4]. Bone is a common site of metastasis and incidence of lung cancer metastasizing to bone is around 36\% [5]. Zoledronic acid, a bisphosphonate is very effective in the treatment of bone metastasis [6]. Adverse effects associated with it include flu-like symptoms, nausea, arthralgia, renal tubular acidosis, hypocalcemia, esophageal irritation, and fatigue. Hepatic dysfunction exclusively due to zoledronic acid is a very rare occurrence with only two case reports so far brought to the light of scientific committee from Europe and China. Until now, no such report has been mentioned from the Indian subcontinent. Henceforth, we are reporting a case of zoledronic acid-induced liver injury.

\section{CASE REPORT}

A 47-year-old male diagnosed as hypertensive came in the month of February 2014 with complaints of low-grade fever, dry cough, and left-side pleuritic chest pain without dyspnea since 1 month. Breath sounds were diminished and other system examinations were normal. Chest X-ray revealed left upper lobe effusion (Fig. 1) and contrastenhanced computed tomography (CECT) chest were suggestive of pleural effusion of infective etiology (Fig. 2). Pleural fluid analysis revealed exudative picture with a predominance of lymphocytes. The patient was started on antitubercular treatment (ATT) empirical therapy in view of effusion and infection, but he was found negative for acid-fast bacilli along with pleural fluid adenosine deaminase levels as $11 \mathrm{mg} / \mathrm{dl}$. Therefore, ATT was stopped after 2 days. CECT-guided lung biopsy confirmed adenocarcinoma lung and tissue was found positive for epidermal growth factor receptor mutation. Bone scan showed multiple metastases. His first chemotherapy cycle was started in March 2014 with cisplatin $125 \mathrm{mg}$ and pemetrexed $850 \mathrm{mg}$ intravenous (IV) along with the IV infusion of $4 \mathrm{mg}$ zoledronic acid. A total of eight cycles were given comprising one cycle once a month and during the course of treatment patient was stable and fine without any symptoms. In September 2014, he complained of severe dyspnea; on examination, massive left-side pleural effusion was noted. Intercostal drain was put, and fluid was drained along with pleurodesis using bleomycin. The patient recovered within few days and chemotherapy was started again with the same regimen. In January-February 2015, routine investigations unfolded the deranged liver functions, ascertained by markedly elevated liver enzymes (Fig. 3 and 4) and zoledronic acid was suspected as the underlying cause of hepatic dysfunction. In February 2015, zoledronic acid was stopped (dechallenge) and never re-administered (rechallenge), gradually patient showed recovery and liver function test (LFT) became normal within a span of 2 months (Fig. 3 and 4).

\section{DISCUSSION}

Lung cancer is one of the most common cancers, accounting for $13 \%$ of all new cancers diagnosed and $19 \%$ as a cause of mortality due to cancer worldwide. In India, lung cancer holds $6.9 \%$ of newly diagnosed cancer with approximately 9.3\% cancer-related deaths [7]. In Asian countries, adenocarcinoma type of lung cancer predominates [7] and various chemotherapeutic regimens are available for treating adenocarcinoma. Pemetrexed containing chemotherapy regimens have gained importance in recent past because of favorable response, improved patient survival and better tolerability over taxanes and another group of drugs [4]. Bone is the common site of metastasis owing to high blood flow in red marrow with primary cancers being prostate, 
breast, kidney and lung [5] and incidence of lung cancer metastasizing to bone is found to be high [5]. Irrespective of whether the bone lesions are osteolytic or osteoblastic; zoledronic acid, a bisphosphonate class of drug is very effective in the treatment of bone metastasis as well in the prevention of metastasis seen with advanced cancers [6].

Bisphosphonates are pyrophosphate analogs; act by binding to the hydroxyapatite and thereby accumulating in the bone and preventing osteoclastic activity. The underlying mechanism of action in bones is it first binds to the surface of bone, then gets adsorbed on mineral

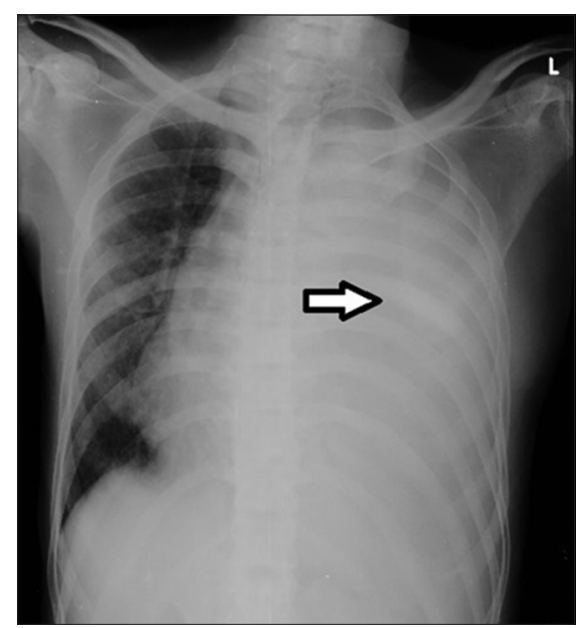

Fig. 1: Chest X-ray showing left upper lobe effusion

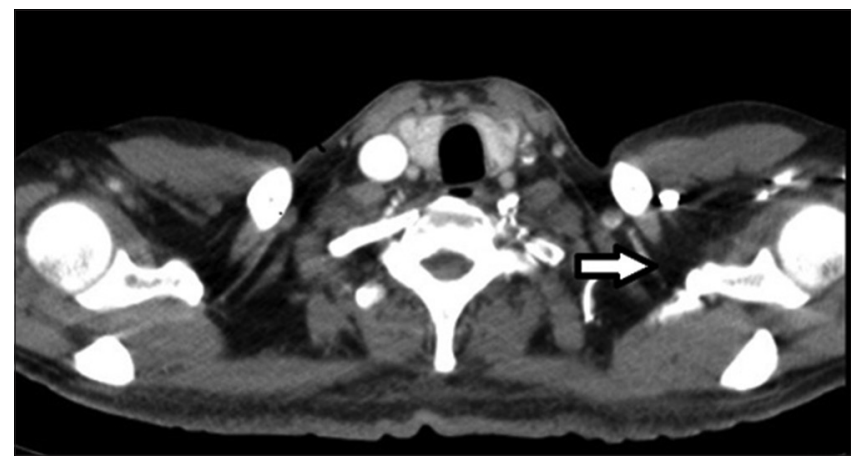

Fig. 2: Contrast-enhanced computed tomography chest suggestive of infective etiology showing pleural effusion surfaces of the bone and later internalizing into osteoclasts, finally leading to disruption of the bone-resorbing activity of osteoclasts [8] In addition, it suppresses osteoclast maturation as well as brings about apoptosis. In a case of metastatic bone lesions, bisphosphonates inhibit cancer cell bone matrix interaction, furthermore will hinder the cancerous invasion of bones [9]; making bisphosphonates the drug of choice to treat both osteolytic and osteoblastic metastatic lesions.

Bisphosphonates have become the popular choice in the past decade to treat postmenopausal, glucocorticoid-induced and transplantassociated osteoporosis, Paget's disease and metastatic bone lesions due to osteotropic cancers [10]. The adverse effect profile of this class of drug includes upper gastrointestinal side effects-esophagitis, esophageal erosions, gastric ulcers; influenza-like symptomsfever, arthralgia, fatigue, bone pain; fragile fractures of atypical nature predominantly involving femur; osteonecrosis of jaw; atrial fibrillations; renal damage-acute tubular necrosis and focal segmental glomerulosclerosis [11]. Whereas, hepatotoxicity is a very rare side effect seen due to bisphosphonates with only a few case reports to support this evidence [12-15]. As per the literature search, hepatic damage solely due to zoledronic acid is an unusual outcome; especially in the absence of any pre-existing liver disease, concomitant medication implicated to cause hepatotoxicity, evidence in favor of zoledronic acid as an attributing factor for hepatic dysfunction is scanty and so far only two case reports have been reported [16,17]. A pre-clinical study carried in rats has shown biopsy-confirmed hepatotoxicity due to zoledronic acid administration [18]. Earlier two case reports have been noted in female patients diagnosed with postmenopausal osteoporosis and Paget's disease, demonstrating the transient rise in liver enzymes. No serious liver injury was implicated [16,17]. Compared to earlier published cases, by our knowledge, it is the first report of zoledronic acid associated hepatic injury in a male patient suffering from cancer. Through literature search displayed no report of zoledronic acid associated liver injury from Indian subcontinent till date.

The mechanism by which zoledronic acid causes hepatotoxicity remains elusive, yet some theories to support this uncommon finding can be stated as follows: First, the bisphosphonates induce acute phase response mediated by active production of interleukin-6, tumor necrosis factor alpha (TNF $\alpha$ ), and pro-inflammatory cytokines from the T-cells and macrophages. These cytokines mainly TNF $\alpha$ has been suspected to explain drug induced-liver injury; TNF $\alpha$ is involved in the activation of various intracellular and apoptotic pathways, which inhibit liver cell proliferation. Moreover, TNF $\alpha$ along with some other pathways - IkB kinase, reactive oxygen species, and stress-activated protein kinases/Jun amino-terminal kinases pathway promotes apoptosis of liver cells $[19,20]$. Second, amino-bisphosphonate like

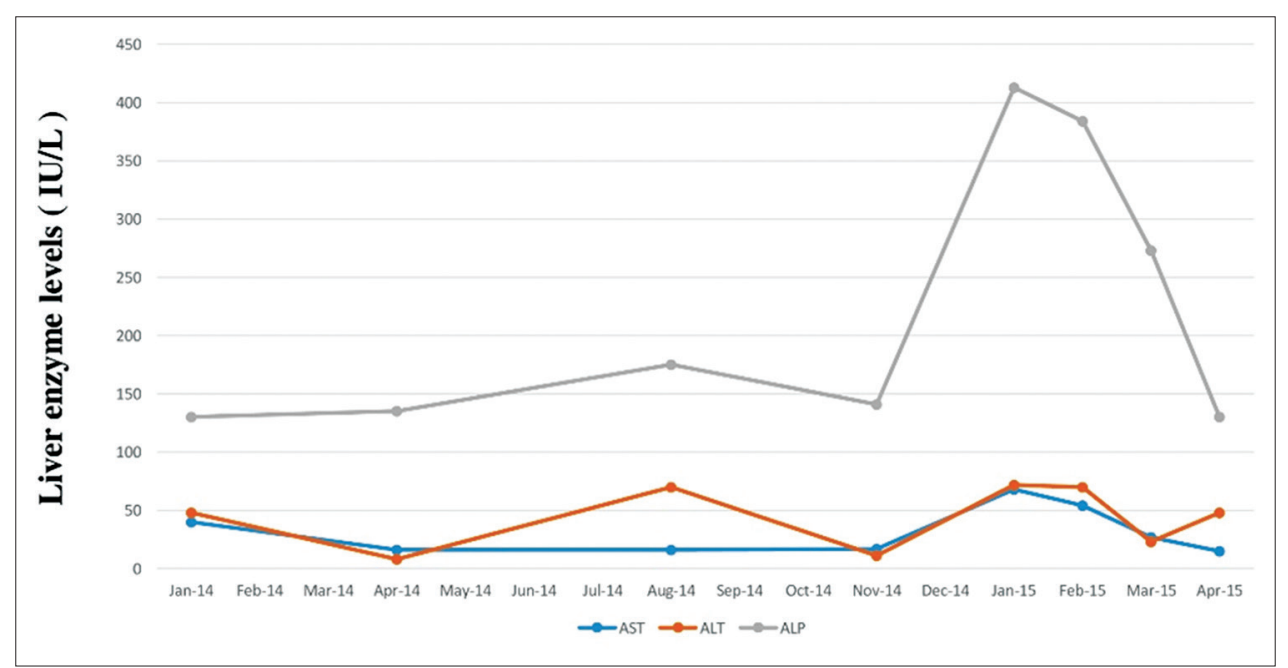

Fig. 3: Depicting the elevated liver enzymes. AST: Aspartate aminotransferase, ALT: Alanine transferase, ALP: Alkaline phosphatase 


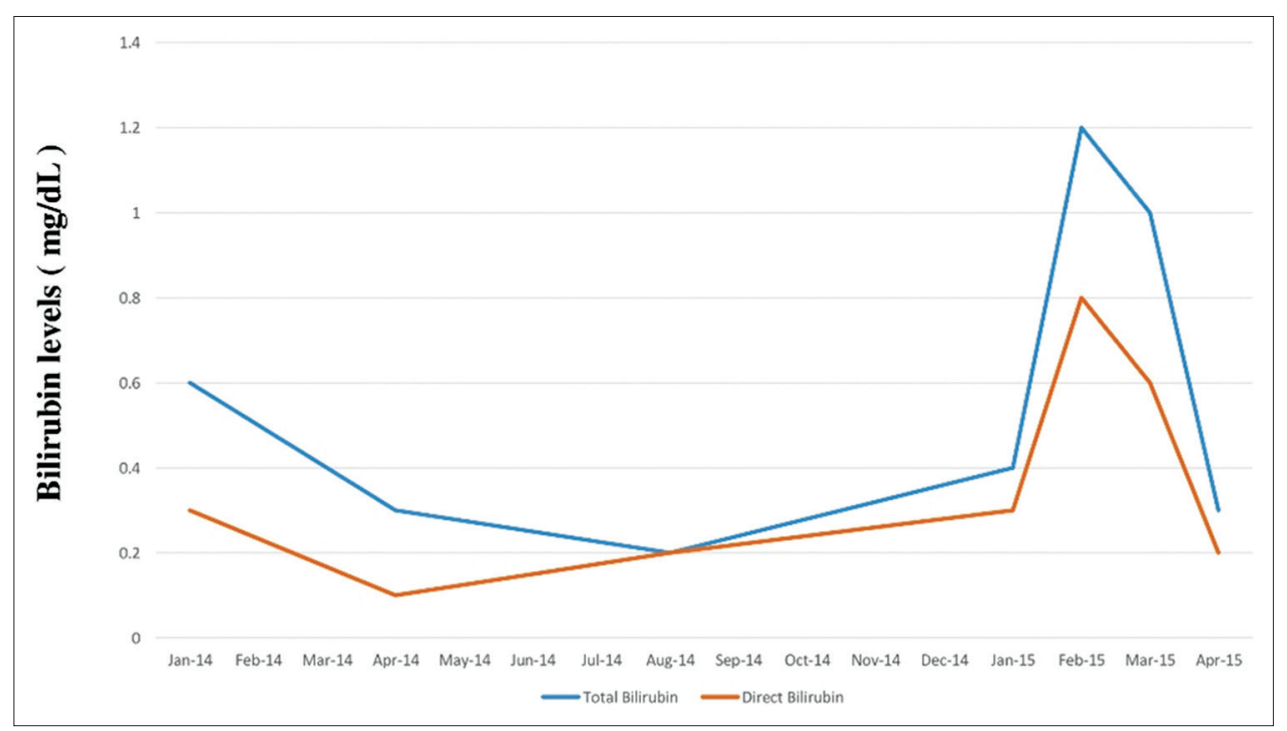

Fig. 4: Depicting the elevated direct bilirubin and total bilirubin

zoledronic acid acts by inhibiting a farnesyl pyrophosphate synthase (FPPS) enzyme, which is responsible for reduced resorptive activity of osteoclast. The suppression of FPPS enzyme leads to un-prenylation of small GTPases such as Rho, Rac, Rab, and Ras; these GTPases regulate vesicle transport, membrane trafficking, cytoskeleton organization and apoptosis under normal physiological conditions. The alteration in protein prenylation might be a contributing factor for liver injury [11]. Third, the zoledronic acid which is nitrogen-containing bisphosphonate has a relatively stronger inhibition of FPPS enzyme compared to other bisphosphonates [11].

In this case, after duration of 10 months of uneventful chemotherapy, there was a sudden elevation in aspartate aminotransferase, alanine transaminase (ALT), alkaline phosphatase (ALP), and direct bilirubin (Figs. 3 and 4) in January 2015. Administration of zoledronic acid was stopped and it was not re-administered; though calcium tablets were continued. Later during follow-up, laboratory investigations exhibited liver enzymes within the normal range. Furthermore, the causal relationship was established using Naranjo's algorithm (score - 6) and Roussel Uclaf Causality Assessment Method (score - 5), displayed a "probable" and "possible" association [21-23]. Furthermore, Hartwig's severity scale $[24,25]$ and Thornton's preventability scale [26] demonstrated adverse drug reaction to being moderately severe and not preventable, respectively. The other risk factors-alcohol use, age $>55$ years, concomitant hepatotoxic drugs, hepatitis B, hepatitis $\mathrm{C}$, a recent history of hypotension, shock or ischemia within past 2 weeks, primary biliary cirrhosis, sclerosing cholangitis were excluded out. Every drug has its own particular mechanism implicated for moreor-less liver injury. In view of less prominent features in favor of liver injury, asymptomatic ALT $\geq 8 \mathrm{~N}$ or a $\geq 1.5$-fold elevated direct bilirubin along with raised ALT levels can be considered as a diagnostic [27]. The greater rise in ALP can be attributed to both bone metastasis and liver injury and it is very hard to differentiate. Neither liver biopsy was done nor rechallenge of zoledronic acid was executed. Moreover, rapid improvement observed in laboratory values of liver enzymes following drug withdrawal raises the susceptibility of drug-induced liver damage. Consensus supports if there is a decline in $>50 \%$ of liver enzymes within 30 days following cessation of suspected drug and it is druginduced injury [28].

\section{CONCLUSION}

Drug-induced hepatotoxicity due to nitrogen-containing bisphosphonates particularly zoledronic acid is a rare occurrence; since a causal relationship was successfully established in this case, hence it will be wise to do continuous monitoring of LFTs during zoledronic acid administration. Every individual drug has its own trademark pattern of liver injury and with only handful previous case reports to support zoledronic acid induced hepatic damage; it should be advised to identify the adverse event early and preventive actions taken soon to delay further progression. If additional studies are done in regard to pharmacokinetic properties of zoledronic acid in genetically predisposed population, it will add a valuable tool to do a confirmatory diagnosis. To sum up, vigilant monitoring along with timely assessment and management can prevent the occurrence of irreversible liver damage.

\section{REFERENCES}

1. Ferlay J, Soerjomataram I, Dikshit R, Eser S, Mathers C, Rebelo M, et al. Cancer incidence and mortality worldwide: Sources, methods and major patterns in GLOBOCAN 2012. Int J Cancer 2015;136(5):E359-86.

2. Ganesh B, Sushama S, Monika S, Suvarna P. A case-control study of risk factors for lung cancer in Mumbai, India. Asian Pac J Cancer Prev 2011;12(2):357-62.

3. Lortet-Tieulent J, Soerjomataram I, Ferlay J, Rutherford M, Weiderpass E, Bray F. International trends in lung cancer incidence by histological subtype: Adenocarcinoma stabilizing in men but still increasing in women. Lung Cancer 2014;84(1):13-22.

4. Al-Saleh K, Quinton C, Ellis PM. Role of Pemetrexed in advanced nonsmall-cell lung cancer: Meta-analysis of randomized controlled trials, with histology subgroup analysis. Curr Oncol 2012;19(1):e9-15.

5. Suva LJ, Washam C, Nicholas RW, Griffin RJ. Bone metastasis: Mechanisms and therapeutic opportunities. Nat Rev Endocrinol 2011;7(4):208-18.

6. Saad F, Gleason DM, Murray R, Tchekmedyian S, Venner P, Lacombe L, et al. Long-term efficacy of zoledronic acid for the prevention of skeletal complications in patients with metastatic hormone-refractory prostate cancer. J Natl Cancer Inst 2004;96(11):879-82.

7. Malik PS, Raina V. Lung cancer: Prevalent trends \& emerging concepts. Indian J Med Res 2015;141(1):5-7.

8. Lipton A. Pathophysiology of bone metastases: How this knowledge may lead to therapeutic intervention. J Support Oncol 2004;2(3):205-13.

9. Boissier S, Magnetto S, Frappart L, Cuzin B, Ebetino FH, Delmas PD, et al. Bisphosphonates inhibit prostate and breast carcinoma cell adhesion to unmineralized and mineralized bone extracellular matrices. Cancer Res 1997;57(18):3890-4.

10. Drake MT, Clarke BL, Khosla S. Bisphosphonates: Mechanism of action and role in clinical practice. Mayo Clin Proc 2008;83(9):1032-45.

11. Abrahamsen B. Adverse effects of bisphosphonates. Calcif Tissue Int 2010;86(6):421-35.

12. Lieverse RJ. Hepatitis after alendronate. Neth J Med 1998;53(2):271-2.

13. Laitinen $\mathrm{K}$, Taube $\mathrm{T}$. Clodronate as a cause of aminotransferase elevation. Osteoporos Int 1999;10(2):120-2.

14. Phillips MB. Risedronate-induced hepatitis. Am J Med 2007;120(3):e1-2. 
15. Goossens N, Spahr L, Rubbia-Brandt L. Severe immune-mediated drug-induced liver injury linked to ibandronate: A case report. J Hepatol 2013;59(5):1139-42.

16. Polyzos SA, Kountouras J, Anastasilakis AD, Litsas I, Kita M, Arsos G, et al. Zoledronic acid-induced transient hepatotoxicity in a patient effectively treated for Paget's disease of bone. Osteoporos Int 2011;22(1):363-7.

17. Lu Y, Pei Y, Shao Y, Yan S, Ma L, Fang F, et al. Hepatotoxicity induced by zoledronic acid in an aged woman with primary osteoporosis. EXCLI J 2013;12:115-7.

18. Dieterle F, Schlotterbeck G, Binder M, Ross A, Suter L, Senn H Application of metabolomics in a comparative profiling study reveals $\mathrm{N}$-acetylfelinine excretion as a biomarker for inhibition of the farnesyl pathway by bisphosphonates. Chem Res Toxicol 2007;20(9):1291-9.

19. Schwabe RF, Brenner DA. Mechanisms of liver injury. I. TNF-alphainduced liver injury: Role of IKK, JNK, and ROS pathways. Am J Physiol Gastrointest Liver Physiol 2006;290(4):G583-9.

20. Papapetrou PD. Bisphosphonate-associated adverse events. Hormones (Athens) 2009;8(2):96-110

21. Naranjo CA, Busto U, Sellers EM, Sandor P, Ruiz I, Roberts EA, et al.
A method for estimating the probability of adverse drug reactions. Clin Pharmacol Ther 1981;30(2):239-45.

22. Danan G, Teschke R. RUCAM in drug and herb induced liver injury: The update. Int J Mol Sci 2015;17. pii: E14.

23. Antony A. Identification and analysis of adverse drug reactions associated with cancer chemotherapy in hospitalized patients. Int J Pharm Pharm Sci 2016;8(7):1-4.

24. Hartwig SC, Siegel J, Schneider PJ. Preventability and severity assessment in reporting adverse drug reactions. Am J Hosp Pharm 1992;49(9):2229-32.

25. Kathiria JM, Sattigeri BM, Desai PM, Patel SP. A study of adverse drug reactions in patients admitted to intensive care unit of a tertiary care teaching rural hospital. Int J Pharm Pharm Sci 2013;5(1):160-3.

26. Schumock GT, Thornton JP. Focusing on the preventability of adverse drug reactions. Hosp Pharm 1992;27(6):538.

27. Andrade RJ, Robles M, Fernández-Castañer A, López-Ortega S, LópezVega MC, Lucena MI. Assessment of drug-induced hepatotoxicity in clinical practice: A challenge for gastroenterologists. World J Gastroenterol 2007;13(3):329-40.

28. Bénichou C. Criteria of drug-induced liver disorders. Report of an international consensus meeting. J Hepatol 1990;11(2):272-6. 